\title{
Moser Vector Fields and Geometry of the Mabuchi Moduli Space of Kähler Metrics
}

\author{
Daniel Guan \\ Department of Mathematics, University of California at Riverside, Riverside, CA 92521, USA \\ Correspondence should be addressed to Daniel Guan; zguan@math.ucr.edu
}

Received 14 June 2013; Accepted 11 October 2013; Published 2 January 2014

Academic Editor: Reza Saadati

Copyright (C) 2014 Daniel Guan. This is an open access article distributed under the Creative Commons Attribution License, which permits unrestricted use, distribution, and reproduction in any medium, provided the original work is properly cited.

\begin{abstract}
There is a natural Moser type transformation along any curve in the moduli spaces of Kähler metrics. In this paper we apply this transformation to give an explicit construction of the parallel transformation along a curve in the Mabuchi moduli space of Kähler metrics. This is crucial in the proof of the equivalence between the existence of the Kähler metrics with constant scalar curvature and the geodesic stability for the type II compact almost homogeneous manifolds of cohomogeneity one mentioned in (Guan 2013). We also explain a new description of the geodesics and prove a curvature property of the moduli space, called curvature symmetric, which makes it similar to some special symmetric spaces with nonpositive curvatures, although the spaces are usually not complete. Finally, we generalize our geodesic stability conjectures in (Guan 2003) and give several results on the Lie algebra structures related to the parallel transformations. In the last section, we generalize the Futaki obstruction of the Kähler-Einstein metrics to the parallel vector fields of the invariant Mabuchi moduli space. We call the related stability the parallel stability. This includes the toric and cohomogeneity one cases as well as the spherical manifolds.
\end{abstract}

\section{Introduction}

In the study of existence of Kähler-Einstein metrics Mabuchi introduced the geodesic equations in [1]. It turns out that the special homogeneous complex Monge-Ampére equation Semmes considered in [2] is exactly the same equation considered by Mabuchi. Let $(M, \omega)$ be a compact Kähler manifold, and let $\varphi(t, m)$ be a real smooth function with variables of time $t$ and point $m \in M$. Regarding $t$ as the real part of a complex number $z_{0}, \varphi$ is a function of $z_{0}$ and $m$. If $\varphi$ satisfies the complex homogeneous Monge-Ampére equation $\operatorname{det}(\omega+\partial \bar{\partial} \varphi)=0$ and $\omega_{t}=\omega+\partial_{M} \bar{\partial}_{M} \varphi$ is a Kähler metric for $t \in[0, T]$, then $\omega_{t}$ is a geodesic in the Mabuchi moduli space of the Kähler metrics.

Locally if $F(0, m)$ is the Kähler potential of $\omega$ and $F(t, m)=F(0, m)+\varphi$, by letting $F\left(z_{0}, m\right)=F(t, m)$ we see that the equation is the same as $\operatorname{det}(\partial \bar{\partial} F)=0$.

It was observed that the kernel of $\partial \bar{\partial} F$ induced a vector field of a certain Moser type transformation. And the Moser type vector field $W$ can be applied to any general curve in the space of the Kähler metrics. In this paper we will apply this Moser type transformation to give an explicit construction for the parallel transformation along any curve in the Mabuchi moduli spaces of Kähler metrics. The existence of the parallel transformation was proven in [1, page 234] by integration of a vector field and then in [3], without knowing Mabuchi's first proof, obtained by solving one of the two quasilinear equations which led to the existence of the geodesics therein. That eventually gave a proof of the opposite direction of $[4,5]$ in 2003 for the cohomogeneity one case. We will give a different proof of the existence of the parallel transformation in this paper. The corresponding quasilinear equation is actually $L_{W} h=0$. Our argument here is independent of [3]. While we dealt mostly with the case with a flat Mabuchi moduli space of Kähler metrics in a given Kähler class in [3], we deal with the general case here.

One can also easily observe that the geodesic equation is actually $L_{W} \dot{F}=0$.

From the properties of the Moser vector fields we also expect that there should be some applications to metrics flows. We will investigate this in the last three sections and in the near future. In particular, we prove that the curvature is parallel on the Mabuchi moduli space of the Kähler metrics of a given Kähler class. 
After the first version of this paper, many things happened. It is evident that, to define a generalization of Kähler metrics with constant scalar curvature, instead of the scalar curvature being a potential function of a holomorphic vector field as Calabi did, we could consider with a Kähler metrics with a scalar curvature being a function as the restriction of a parallel function on the equivariant Mabuchi moduli space of Kähler metrics. This is motivated by [6]. For example, in the case of cohomogeneity one with a semisimple automorphism group in $[7,8]$, the Futaki invariants are zero. Therefore, there are no Calabi extremal metrics in general. However, there are nontrivial parallel functions on the equivariant Mabuchi moduli space and therefore, there are many "generalized" Calabi extremal metrics; see also [9]. In particular, any equivariant Kähler metric is a generalized Calabi extremal metric in this sense. Of course, we need more restriction to get a more meaningful generalized Calabi extremal metric. In [10] in 2001, it was proven that Calabi flow was better than the generalized Ricci flow in general. But it seemly was only true for the low dimensional case. When we tried to publish [10], we proposed that the Calabi flow always has the long time existence property just as what Cao and Koiso observed in the Kähler-Ricci flow. However, it seems possible that it is only true for the low dimensional case. For example, in the case of $\mathbf{C} P^{n}$ with the standard cohomogeneity one metrics as in [10], as one end has a high codimension $n$, the Calabi flow might not always have long time existence. Therefore, it is easier to consider a generalized Calabi metric called the $m$ extremal metric and the related $m$-Calabi flow [11]. To make the short time existence easier, one might actually consider an exponential Calabi extremal metric or e-extremal metric and $e$-Calabi flow.

\section{Preliminaries}

Definition 1. Let $\omega_{t}$ be a family of symplectic forms. If a vector field $X(t, m)$ satisfies $X\left(\omega_{t}\right)=0$, then we call $X$ a Moser vector field.

See also Moser's original paper [12].

Regarding $\partial \bar{\partial} F=\left(F_{i \bar{j}}\right)$ as a matrix, let $\left(A_{i \bar{j}}\right)$ be the adjoint matrix; then $F_{i \bar{j}} A_{j \bar{k}}=\delta_{i k} \operatorname{det}(\partial \bar{\partial} F)$ for any $i, k \in(0,1, \ldots, n)$. Applying this formula we obtain the following.

Lemma 2. $Y=A_{0 \overline{0}}\left(\partial / \partial z^{0}\right)+A_{0 \bar{s}}\left(\partial / \partial z^{s}\right)$ is in the kernel if $\operatorname{det}(\partial \bar{\partial} F)=0$.

Let $W=\operatorname{Re}\left(Y / A_{0 \overline{0}}\right)$. In general, we let $\omega_{t}=\left(\omega_{t, i \bar{j}}\right)=$ $\omega_{0}+\partial \bar{\partial} \varphi_{t}$ be a curve in the space of Kähler metrics even if we do not have $\operatorname{det}(\partial \bar{\partial} F)=0$, and we can define $Y$ and $W$ in the same way with $F_{t}=F_{0}+\varphi_{t}$. Then by direct calculation we obtain the following.

Lemma 3. We have $\left.L_{W}\left(\partial_{M} \bar{\partial}_{M} F\right)\right|_{M}=0$.

Proof. Let $X=Y / A_{0 \overline{0}}$ and denote $\partial_{i}=\partial / \partial z^{i}, \partial_{\bar{i}}=\partial / \partial \bar{z}^{i}$. Then

$$
L_{X}=L_{\partial_{0}}+L_{\left(A_{0 \bar{s}} / A_{0 \overline{0}}\right) \partial_{s}} \cdot
$$

By

$$
\begin{aligned}
L_{a_{i} \partial_{i}}\left(d z^{k}\right)\left(\partial_{l}\right) & =L_{a_{i} \partial_{i}}\left(\delta_{k l}\right)-d z^{k}\left(\left[a_{i} \partial_{i}, \partial_{l}\right]\right) \\
& =d z_{k}\left(\partial_{l}\left(a_{i}\right) \partial_{i}\right)=\partial_{l}\left(a_{k}\right) \\
& =a_{k, l},
\end{aligned}
$$

similarly we have

$$
L_{a_{i} \partial_{i}}\left(d z^{k}\right)\left(\partial_{\bar{l}}\right)=a_{k, \bar{l}}
$$

that is, $L_{a_{i} \partial_{i}}\left(d z^{k}\right)=a_{k, l} d z^{l}+a_{k, l} d \bar{z}^{l}$. Similarly, we have $L_{a_{i} \partial_{i}} d \bar{z}^{k}=0$.

Combining this together with the identity

$$
F_{0 \bar{m}} A_{0 \overline{0}}+F_{r \bar{m}} A_{0 \bar{r}}=0
$$

and its differentiations with respect to $z_{s}$ and $\bar{z}_{s}$, we finally obtain

$$
\begin{aligned}
&\left.L_{X}\left(F_{s \bar{m}} d z^{s} \wedge d \bar{z}^{m}\right)\right|_{M} \\
&=\left(\dot{F}_{s \bar{m}}+\frac{A_{0 \bar{r}}}{A_{0 \overline{0}}} F_{s \bar{m} r}+F_{r \bar{m}}\left(\frac{A_{0 \bar{r}, s}}{A_{0 \overline{0}}}-\frac{A_{0 \bar{r}} A_{0 \overline{0}, s}}{A_{0 \overline{0}}^{2}}\right)\right) \\
& \quad \times d z^{s} \wedge d \bar{z}^{m} \\
& \quad+F_{r \bar{m}}\left(\frac{A_{0 \bar{r}}}{A_{0 \overline{0}}}\right)_{\bar{s}} d \bar{z}^{s} \wedge d \bar{z}^{m} \\
&=\frac{-A_{0 \overline{0}, s}}{A_{0 \overline{0}}^{2}}\left(\dot{F}_{\bar{m}} A_{0 \overline{0}}+F_{r \bar{m}} A_{0 \bar{r}}\right) d z^{s} \wedge d \bar{z}^{m} \\
&-\left(F_{r \overline{m s}} \frac{A_{0 \bar{r}}}{A_{0 \overline{0}}}+\dot{F}_{\bar{m} \bar{s}}\right) d \bar{z}^{m} \wedge d \bar{z}^{s}=0
\end{aligned}
$$

as required.

Proposition 4. $W$ induces a family of Moser differential transformations $T(t)$ such that $T\left(\left.\partial_{M} \bar{\partial}_{M} F\right|_{M}\right)=\left.\partial_{M} \bar{\partial}_{M} F\right|_{M}$.

By the parallel transformation formula in $[1,3,6]$, we have the following.

Lemma 5. A function $h$ is a parallel transformation, regarded as a vector field in the moduli space of Kähler metrics, along a path $\omega_{t}=\omega_{0}+\partial_{M} \bar{\partial}_{M} \varphi$ if and only if $\dot{h}-(1 / 2)(d h, d \dot{F})=0$.

\section{Existence of the Parallel Transformations}

In general, let $\omega_{t}=\left(\omega_{t, i \bar{j}}\right)=\omega_{0}+\partial \bar{\partial} \varphi_{t}$ be a curve in the space of Kähler metrics (might not be a geodesic curve) we see that;

$$
A_{0 \overline{0}}\left(\dot{h}-\frac{1}{2}(d h, d \dot{F})\right)=\operatorname{Re} A_{0 i} h_{i}=A_{0 \overline{0}} L_{W} h .
$$

Therefore, we have the following.

Theorem 6. $h$ is parallel if and only if $L_{W} h=0$. In particular, $\omega_{t}$ is a geodesic if and only if $L_{W} \dot{F}=0$. 
Once we have a curve in the moduli space of Kähler metrics we have the orbits of the Moser vector field $W$. Let $h_{0}$ be any function on $M$; regarding the initial vector field at $\omega_{0}$, we can let $h$ be constant along the orbits. Then $h$ is parallel along this given curve in the moduli space. In this way, we have an explicit construction of the vector field $h$ from the initial value $h_{0}$. Therefore, we have the following.

Corollary 7. Given any curve $C: \omega_{t}$ with $t \in[0,1]$ in the moduli space of the Kähler metrics in a Kähler class and an initial vector $h_{0}$ at $\omega_{0}$, there is a unique smooth parallel vector field along $C$.

In [3], without knowing the first proof of Mabuchi in [1, page 234], we had used Corollary 7 to find global parallel vector fields on the compact complex almost homogeneous Kähler manifolds with algebraic reductive groups and to obtain parallel coordinates. Then by integrating the global parallel vector fields we will obtain many smooth geodesics.

\section{Poisson Bracket and the Curvature Symmetric Property of the Mabuchi Moduli Space of the Kähler Metrics in a Given Kähler Class}

For a given Kähler structure we have a symplectic structure. Therefore, there is a Poisson structure $\{f, g\}=\omega^{*}(d f, d g)$ or $d f \wedge d g \wedge \omega^{n-1}=\{f, g\} \omega^{n}$. By identifying $\{f \in$

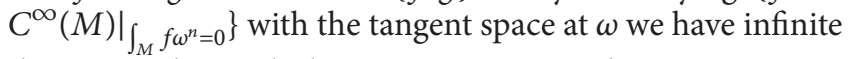
dimensional Lie algebra structure on each tangent space. It is actually an infinite dimensional Lie algebra with an invariant metric (more like compact Lie algebra of the finite dimensional case; see [13]). Now we would like to see some more global pictures.

Let $\phi=\phi(t)$ be a curve in the Mabuchi moduli space, and let $\varphi_{i}$ be two parallel vector fields along $\phi$ for $i=1,2$.

Then in view of Lemma 3 and Proposition 4, identifying by the Moser transformation we can consider the family of Kähler structures as the same symplectic structure, and $\varphi_{i}$ being regarded as functions of this symplectic manifold are independent of $t$ by Theorem 6. The Poisson bracket $\left\{\varphi_{1}, \varphi_{2}\right\}$ also does not depend on $t$ and therefore is parallel along the curve of the Kähler structures. This parallelism can be expressed as follows.

Theorem 8. We have

$$
\frac{d}{d t}\left(\left\{\varphi_{1}, \varphi_{2}\right\}\right)=\frac{1}{2}\left(d \dot{\phi}, d\left\{\varphi_{1}, \varphi_{2}\right\}\right) .
$$

Therefore, by the formula of the curvature in [1] (see also $[2,14]$, [15, page 195 Theorem A]) we have that

$$
R(f, g) h=-\frac{1}{4}\{\{f, g\}, h\}
$$

we also have that the curvature is constant along the curve by the curvature formula.

Theorem 9. On the moduli space $\nabla R=0$.
We call an infinite dimensional Riemannian manifold curvature symmetric if $\nabla R=0$. Theorem 14 just says that the Mabuchi moduli space of the Kähler metrics in a given Kähler class is curvature symmetric.

Therefore, the moduli space has a quite good structure. However, we see in [7] that the moduli space, in general, is incomplete. This gives a negative answer to an expectation of Semmes [2] and Donaldson [14] that the moduli space is an infinite dimensional kind of (complete) symmetric space.

On the other hand, our result in this section does give some good properties similar to a convex region (see [15]) in a special symmetric space of nonpositive curvature (cf. [16]).

I personally do believe that the Mabuchi moduli space of the Kähler metrics is locally symmetric; that is, at each point there is a local isometric map which fixes the given point and induces a map of -1 on the tangent space.

\section{The Fourth Geodesic Principle and the Geodesic Stability Conjectures}

When the curve is an infinite geodesic ray, we can obtain a good picture for the general geodesic stability provided that there is enough smoothness. Instead of using parallel geodesics, which works for the case with the moduli space being flat as in [7], we could use infinite geodesic rays with the same point at infinity. This was also pointed out in [10, Remark 4]. It is just as in the case of finite dimensional symmetric spaces. For example, two maximal geodesic rays $\gamma_{1}$ and $\gamma_{2}$ with $d\left(\gamma_{1}(t), \gamma_{2}(t)\right)<C$ for some positive constant $C$ go to the same point at infinity. One can use a finite dimensional nonpositively curved symmetric space as a model, for example, the Poincare metric on the upper half plane $\operatorname{Im} z>0$.

In the case with the Poincare metric, the geodesics are straight lines perpendicular to the $x$-axis and the half circles with the centers on the $x$-axis. Two geodesic rays go to the same point at infinity if their end points on the $x$-axis are the same.

Now let us go back to the infinite dimensional picture on the Mabuchi moduli space. Along each maximal geodesic ray, since $\nabla R=0$, we can have a frame of vector fields such that the sectional curvatures are constant and nonpositive. The corresponding Jacobi field of family of geodesics going to the same point at infinity has the form

$$
J=\sum_{i=0}^{+\infty} a_{i} e^{-b_{i} t} \varphi_{i}
$$

where $\varphi_{i}$ are parallel along the geodesic and $b_{0}=0<b_{1}<$ $\cdots<b_{i}<b_{i+1}<\cdots$ assuming that there exists an orthogonal basis with respect to both the metric and the curvature along the given geodesic (notice that this is not true in general). See [17, page 15] and notice a sign difference for the curvature. The above formula will be $J=\sum_{i=1}^{N} a_{i} e^{-b_{i} t} \varphi_{i}$ for the finite dimensional case and we just replace $N$ by $+\infty$ here. We had dealt with some flat cases in [3] in which $a_{i}=0$ for $i>0$.

Let us have another way to understand this. Let $\mathscr{M}_{K}$ be the Mabuchi moduli space of the invariant Kähler metrics with respect to a maximal compact subgroup $K$ of the complex 
automorphism group. Rescale it by a sequence of positive numbers $A_{i} \rightarrow 0$. Recall that on the tangent space at $\omega$ of the Mabuchi moduli space, the Riemannian metric is

$$
g\left(\phi_{1}, \phi_{2}\right)=\int_{M} \phi_{1} \phi_{2} \omega^{n}
$$

We let $g_{A_{i}}=A_{i} g$ and fix a Kähler metric $\omega$. We denote the corresponding marked Riemannian manifold by $A_{i}\left(\mathscr{M}_{K}, \omega\right)$. Assuming that we can have an exponential map, we identify $A_{i}\left(\mathscr{M}_{K}, \omega\right)$ as a subset of $\left(\mathscr{M}_{K}, \omega\right)$ by the differentiable map

$$
j_{A_{i}}=\exp _{\left(\mathscr{M}_{K}, \omega\right)} \cdot i_{A_{i}} \cdot \exp _{A_{i}\left(\mathscr{M}_{K}, \omega\right)}^{-1},
$$

where exp are the exponential maps at $\omega$ and $i_{A_{i}}$ is the natural identity map from the tangent space of $A_{i}\left(\mathscr{M}_{K}, \omega\right)$ to that of $\left(\mathscr{M}_{K}, \omega\right)$. Then, for any point $\omega$, the marked infinite dimensional Riemannian manifolds $A_{i}\left(\mathscr{M}_{K}, \omega\right)$ converge as subsets to a cone $\mathscr{C}$ (rather than a sequence of Riemannian manifolds) with $\omega$ as the vertex point. The structure of $\mathscr{C}$ depends on the choice of $\omega$. Any other point $\omega_{1}$ also converges to the vertex point. The condition that an infinite geodesic ray $\gamma_{1}$ from $\omega_{1}$ goes to the same point at infinity as that of a given infinite geodesic ray $\gamma$ from $\omega$ is defined as $\lim _{i \rightarrow+\infty} \gamma_{1}=$ $\lim _{i \rightarrow+\infty} \gamma$.

A general Jacobi field on $\left(\mathscr{M}_{K}, \omega\right)$ has the form

$$
J=\left(a_{0}^{1} \varphi_{0}^{1}+a_{0}^{2} t \varphi_{0}^{2}\right)+\sum_{n=1}^{+\infty}\left(a_{n}^{1} e^{-b_{n} t} \varphi_{n}^{1}+a_{n}^{2} e^{b_{n} t} \varphi_{n}^{2}\right)
$$

where $a_{n}^{k}$ are constants and $\varphi_{n}^{k}$ are parallel functions for $k=$ 1,2 (or $a_{n}^{2}$ are zeros except finite of them).

For $A_{i}\left(\mathscr{M}_{K}, \omega\right)$, we have $t_{i}=A_{i} t$ and $A_{i} \varphi_{n, i}^{k}=\varphi_{n}^{k}$. Therefore,

$$
\begin{aligned}
J_{i}= & \left(a_{0}^{1} A_{i} \varphi_{0, i}^{1}+a_{0}^{2} t_{i} \varphi_{0, i}^{2}\right) \\
& +\sum_{n=1}^{+\infty}\left(A_{i} a_{n}^{1} e^{-A_{i}^{-1} b_{n} t_{i}} \varphi_{n, i}^{1}+A_{i} a_{n}^{2} e^{A_{i}^{-1} b_{n} t_{i}} \varphi_{n, i}^{2}\right) .
\end{aligned}
$$

Therefore, formally we can let

$$
J_{\infty}=a_{0}^{2} t_{\infty} \varphi_{0, \infty}+\sum_{n=1}^{+\infty} a_{n}^{2} e^{b_{n} t_{\infty}} \varphi_{n, \infty} .
$$

Other terms tend to zero. Let $M\left(\omega_{1}, \omega_{2}\right)$ be the Mabuchi functional. For any maximal geodesic ray $\gamma$, we let

$$
F(\gamma)=\lim _{t \rightarrow+\infty} \frac{d M}{d t}(\gamma(0), \gamma(t))
$$

We then have the following.

Conjecture 10. Two infinite geodesic rays $\gamma_{1}$ and $\gamma_{2}$ have the same generalized Futaki invariant if $\lim _{i \rightarrow+\infty} \gamma_{1}=$ $\lim _{i \rightarrow+\infty} \gamma_{2}$.

The structure of the Jacobi field strongly supports this conjecture. We already have three geodesic stability principles in [7]; we might call Conjecture 10 the geodesic stability principle 4.

This is exactly what we have in Remark 4 of [10]. But we have more detailed picture here.

Let $\mathscr{C}_{1}$ be the subcone of $\mathscr{C}$ of all incomplete maximal geodesic rays, that is, those only parametrized on half lines; as in [7] we have the following.

Conjecture 11. If we identify $\mathscr{C}_{1}$ as part of the tangent space at a given Kähler metric $\omega_{0}$, then there is an extremal metric if and only if the generalized Futaki invariants of maximal geodesic rays are positive and bounded from blow by a given seminorm.

As in $[6,7]$, by the convex property of the modified Mabuchi functional along the geodesics we see that the generalized Futaki invariant cannot be $-\infty$. The generalized Futaki invariant of a maximal geodesic ray might be $+\infty$. We have the following.

Conjecture 12. The subset $\mathscr{C}^{\prime}$ of maximal geodesic rayc $\in \mathscr{C}_{1}$ with finite generalized Futaki invariant is a subcone and $F(c)$, $c \in \mathscr{C}^{\prime}$, is linear on $\mathscr{C}^{\prime}$ with respect to $J_{\infty}$.

Then we have the following.

Conjecture $11^{\prime}$. A Kähler class has an extremal metric if and only if $F(c) \geq|c|>0, c \in \mathscr{C}^{\prime}$ for a given seminorm $|\cdot|$.

We remark here that we need a seminorm here; that is, $F(c)>0, c \in \mathscr{C}_{1}$, is not enough as we see in [7] and the Ding-Tian type generalized Futaki invariant therein (cf. [18]) might not come from an $F(c)$ as in [7]. We still need to understand what the given seminorm is. However, our picture of the existence of the extremal metrics is quite clear now.

Remark 13. To convince the readers that the geodesic stability is the right stability we should give a philosophical "proof" for the Kähler-Einstein case. If we consider the Kähler-Ricci flow, Perelman's estimate, also earlier by Cao and Koiso, shows that the change of the metric is bounded. If a subsequence of the metrics converges to a metric of a finite distance, even a singular metric, then, by the first and the second geodesic stability principles in [7] (see also [8] for updated version), it has a finite Mabuchi functional and the sequence of the Kähler metrics converges to a Kähler-Einstein metric. If there is not any finite limit, by the first stability principle there is a unique geodesic connecting $g_{0}$ and $g_{t}$. We call it $\gamma_{t}$. Extend the $\gamma_{t}$ to be a maximal geodesic ray. Let $t_{i}$ be a series of $t$ such that $\gamma_{t_{i}}$ converges to a possibly singular maximal geodesic ray $\gamma$. Then the stability condition in the third geodesic stability principle (or our Conjecture 11 here) would imply that the Mabuchi functional would increase along $\gamma$ eventually, a contradiction.

\section{Global Parallel Vector Fields}

Moreover, by [6] the potential functions of the vector fields of the Lie algebra $\mathscr{K}$ of $K$ are parallel on the moduli space of the invariant metrics. In particular, the vector potential functions of elements of the center $\mathscr{T}$ of the Lie algebra $\mathscr{K}$ of $K$ also induce families of invariant Kähler metrics. Notice that no 
all the potential functions of Lie algebra in the tangent space of the moduli space of the invariant Kähler metrics-they are in the tangent space the bigger (nonnecessary invariant) moduli space. And it is natural for us to restrict our attention to the smaller moduli space. Therefore, these functions from the center are in the tangent space of the Mabuchi moduli space of the invariant Kähler metrics and are parallel. Thus, there might be some global parallel vector fields on $\mathscr{M}_{K}$. We denote the set of all the global parallel vector fields by $\mathscr{P}$. Then by Theorems 6 and 8 , we have the following.

Theorem 14. Let $f_{1}, \ldots, f_{k} \in \mathscr{P}$; then, for any analytic function $F\left(x_{1}, \ldots, x_{k}\right)$, the composition function $F\left(f_{1}, \ldots, f_{k}\right)$ is parallel. Moreover, the set of all these parallel vector fields is closed under the Poisson bracket.

For these parallel vector fields we can obtain the geodesics by the method of Section 3. However, as what happened for the toric varieties in [7], unlike those in $\mathscr{T}$, the maximal geodesic might be incomplete and might actually be finite.

Moreover, for any $f_{1}, f_{2} \in \mathscr{P}$, starting from any invariant metric, one should get a 2-dimensional net of geodesics $P_{2}$. If we can prove this, then $P_{2}$ is a geodesic submanifold of $\mathscr{M}_{K}$. Since both $f_{1}, f_{2}$ are parallel on $P_{2}$, we expect that the curvature is zero. We have the following.

Theorem 15. For any $g,\{g, f\}=0$ if $f \in \mathscr{P}$. In particular, the Poisson structure on $\mathscr{P}$ is trivial; that is, $\mathscr{P}$ is abelian.

Proof. Let $\omega$ be any equivariant Kähler metric, and let $g$ be any function in the equivariant tangent space, $f \in \mathscr{P}$, and let $\omega(s, t)$ be a 2-parameter family of Kähler metrics such that $\omega(0,0)=\omega$ and $\omega(s, 0)$ has tangent $g$ and $\omega(0, t)$ has tangent $f$. Let $f \in \mathscr{P}$; then $R(g, f) f=\left(\nabla_{g} \nabla_{f}-\nabla_{f} \nabla_{g}\right) f=0$. Since $R(g, f) f=-(1 / 4)\{\{g, f\}, f\}$ (see [15, page 195]) we get $\{\{g, f\}, f\}=0$ and hence $(R(g, f) f, g)=0$; that is, $\int\{g, f\}^{2} \omega^{n}=0$. Therefore, $\{g, f\}=0$ for any tangent vector $g$.

In particular, if $g \in \mathscr{P}$ also, we have that $\mathscr{P}$ is abelian.

Now we see that $\mathscr{P}$ is in a way similar to $\mathscr{T}$. But sometimes $\mathscr{P}$ is bigger than the algebra of functions generated by $\mathscr{T}$. For the examples in $[3,7], \mathscr{T}=0$ but $\mathscr{P}$ is generated by the function $U$ there. Let $k=\left.\sup _{m \in M} \operatorname{dim} d \mathscr{P}\right|_{m}$; then, at a generic point $m \in M$, we can have $k$ functions $f_{i} \in \mathscr{P}$ which are independent. We call $f_{1}, \ldots, f_{k}$ a parallel coordinate at $m$ with regarding $M$ as a symplectic manifold.

Theorem 16. The $L^{2}$ closure of the infinite-dimensional Lie algebra of equivariant functions $T_{K}=T_{\mathscr{M}_{K}}$ splits as an orthogonal direct sum of the closure of $\mathscr{P}$ and the closure of another infinite-dimensional Lie subalgebra $\left\{T_{K}, T_{K}\right\}$.

Proof. We want to prove that $f \in T_{K}$ is perpendicular to $\left\{T_{K}, T_{K}\right\}$ if and only if $f \in \mathscr{P}$. We have

$$
\int_{M} f\{g, h\} \omega^{n}=0
$$

for any $g, h \in T_{K}$ if and only if $\int_{M}\{h, f\} g \omega^{n}=0$ for any $g \in T_{K}$ with any given $h \in T_{K}$; that is, $\{h, f\}=0$ for any $h \in T_{K}$. In the proof of our Theorem 16 we already see that if $f \in \mathscr{P}$, then $\{h, f\}=0$. We can also prove the other direction. If $\{h, f\}=0$ for any $h \in T_{K}$, then $R(g, h) f=0$. We want to prove that $f$ can be globally defined as a parallel vector field on $\mathscr{M}_{K}$. We notice that the Lie algebra $\left\{T_{K}, T_{K}\right\}$ is invariant under the parallel transformation and hence so is the function orthogonal to $\left\{T_{K}, T_{K}\right\}$. Let $\omega(s, t)$ be a family of curves in $\mathscr{M}_{K}$ and $\omega(s, 0)=\omega_{0}, \omega(s, 1)=\omega_{1}$. Let $f(s, t)$ be family of functions on $M$ such that $f(s, 0)=f$ and $f_{s}(t)$ is parallel along the curves $C_{s}(t)=\omega(s, t)$. Then $f(s, t)$ are orthogonal to $\left\{T_{K}, T_{K}\right\}$. Therefore, $R(g, h) f(s, t)=0$ for any $g, h \in T_{K}$. Now we want to prove that $f(s, 1)$ does not depend on $s$. Since $f$ is parallel along each $\omega_{s}$ we have $\nabla_{t} f=0$; then

$$
\nabla_{t} \nabla_{s} f=\left(\nabla_{t} \nabla_{s}-\nabla_{s} \nabla_{t}\right) f=R\left(f_{t}, f_{s}\right) f=0 ;
$$

that is, $\nabla_{s} f$ is also parallel along each $\omega_{t}$. Since $\nabla_{s} f=0$ at $t=0$, we always have $\nabla_{s} f=0$. In particular, $\nabla_{s} f=$ 0 at $t=1$; that is, $f(s, 1)$ is independent of $s$. Therefore, $f$ can be extended as a global parallel vector field; that is, $f \in \mathscr{P}$.

Now, for any $f \in T_{K},(f, g) g \in \mathscr{P}$ defines a functional on the closure of $\mathscr{P}$. But for the property of a Hilbert space we have a $f_{1}$ in the closure of $\mathscr{P}$ such that $\left(f_{1}, g\right)=(f, g)$ for any $g$ in the closure of $\mathscr{P}$. Let $f_{2}=f-f_{1}$; then $f_{2}$ is in the closure of $\left\{T_{K}, T_{K}\right\}$ and $f=f_{1}+f_{2}$.

For those maximal geodesic rays generated by the functions in $\mathscr{P}$ we already applied our generalized Futaki invariants in [7]. If $M$ is a compact spherical almost homogeneous manifold under an algebraic reductive group, that is, the $K$ invariant functions are generated by an abelian ideal, then $T_{K}=\mathscr{P}$, for example, when $M$ is cohomogeneity one. In that case, any tangent vector at a point can be extended to a global parallel vector field. In these cases, we can apply Mabuchi's definition to define the Futaki invariants for the maximal geodesic rays. The existence of the Kähler-Einstein metrics should imply the positivity of the Futaki invariant for those geodesics not coming from any holomorphic vector field. Even for the toric manifolds, this gives new obstructions since the function might be a function of the potential functions of holomorphic vector fields but itself is not a potential function of any holomorphic vector field. That is exactly what we did in [7]. Also, for these manifolds, the invariant Mabuchi moduli space is flat. In general, even though not all the tangent vectors in the tangent space of the invariant Mabuchi moduli space come from parallel vector fields, we can use the parallel ones to get new obstructions just like the original Futaki obstruction. We call the related stability parallel stability; then we expect that for the cohomogeneity one and the spherical cases including the toric manifolds the existence is the same as the parallel stability.

We can actually expect that the direct sum can be realized as $C^{\infty}$ functions.

Conjecture 17. We have $T_{K}=\mathscr{P}+\left\{T_{K}, T_{K}\right\}$.

While Conjectures $10,11,12$, and $\left(11^{\prime}\right)$ might be very difficult to be proved, Conjecture 17 would be easier. We should try to prove Conjecture 17 in the near future. 
Remark 18. When the group $G$ is Hamiltonian (this is true whenever the manifold is simply connected, e.g., when $M$ is Fano), that is, every element in the Lie algebra $\mathscr{G}$ of $G$ corresponds to a given function, from (3) and (4) of [13, page 3362], one could easily see that $\mathscr{P}$ is generated by the functions related to the Lie algebra of the torus which is the complement of $H$ in $J$. We notice here that $G$ is compact and $J$ is locally a direct product of a torus and $H$. And the functions on the symplectic reduction produce the major part of $\left\{T_{K}, T_{K}\right\}$. To make the picture clearer for the reader, let $\Phi: M \rightarrow \mathscr{G}^{*}$ be the moment map with $\Phi(m)(g)=g(m)$. On each generic orbit $G / H$, we have the moment map $\Psi=\left.\Phi\right|_{G / H}: G / H \rightarrow$ $G / J$. The codimension of $G / J=\Psi(G / H)$ is the same as the dimension of the torus $J / H$. Therefore, $\Psi(M) / / G$ has a dimension of that of $J / H . \mathscr{P}$ is the pullback of the functions on $\Psi(M) / / G$ and the symplectic reduction $M_{G}$ gives the major part of $\left\{T_{K}, T_{K}\right\}$. That is, locally $T_{K}$ is the functions on $\Psi(M) / / G \times M_{G}$. This basically gives a proof for Conjecture 17 .

Remark 19. In the case of [7], one could easily calculate that $\theta$ is the square of the norm of $\Psi(m)$ under the standard product metric of $\mathbf{C} P^{n} \times \mathbf{C} P^{n}$. Therefore, the name phase angle (or square phase angle) is justified. Similarly, $U$ is the square of the norm of $\Psi(m)$ for the corresponding metric.

\section{Conflict of Interests}

The author declares that there is no conflict of interests regarding the publication of the paper.

\section{Acknowledgment}

This work was supported by NSF DMS-0103282.

\section{References}

[1] T. Mabuchi, "Some symplectic geometry on compact Kähler manifolds I," Osaka Journal of Mathematics, vol. 24, pp. 227-252, 1987.

[2] S. Semmes, "Complex monge-ampére and symplectic manifolds," American Journal of Mathematics, vol. 114, pp. 495-550, 1991.

[3] D. Guan, "Jacobi fields and geodesic stability," in preparation.

[4] D. Guan, "Existence of extremal metrics on almost homogeneous manifolds of cohomogeneity one. II," Journal of Geometric Analysis, vol. 12, no. 1, pp. 63-79, 2002.

[5] D. Guan, "Affine compact almost-homogeneous manifolds of cohomogeneity one," Central European Journal of Mathematics, vol. 7, no. 1, pp. 84-123, 2009.

[6] D. Guan, "On modified Mabuchi functional and Mabuchi moduli space of Kähler metrics on toric bundles," Mathematical Research Letters, vol. 6, no. 5-6, pp. 547-555, 1999.

[7] D. Guan, "Existence of extremal metrics on almost homogeneous manifolds of cohomogeneity one. III," International Journal of Mathematics, vol. 14, no. 3, pp. 259-287, 2003.

[8] D. Guan, "Type I compact almost homogeneous manifolds of cohomogeneity one. III," Pacific Journal of Mathematics, vol. 261, no. 2, pp. 369-388, 2013.

[9] D. Guan, "M-extreme metrics and $\mathrm{m}$-Calabi ows," in preparation.
[10] D. Guan, "Extremal solitons and exponential $C^{\infty}$ Convergence of the modified calabi flow on certain $C P^{1}$ bundles," Pacific Journal of Mathematics, vol. 233, no. 1, pp. 91-124, 2007.

[11] D. Guan, "Positive lemmas, generalized extremal-solitons and second order linear differential equations," Advancement and Developement in Mathematical Science, vol. 1, pp. 13-32, 2012.

[12] J. Moser, "On the volume elements on a manifold," Transactions of the American Mathematical Society, vol. 120, pp. 286-294, 1965.

[13] D. Guan, "On compact symplectic manifolds with lie group symmetries," Transactions of the American Mathematical Society, vol. 357, no. 8, pp. 3359-3373, 2005.

[14] S. K. Donaldson, "Symmetric spaces, Kähler geometry and hamiltonian synamics," AMS Translation, vol. 196, no. 2, pp.1333, 1999.

[15] X. X. Chen, “Space of Kähler metrics," Journal of Differential Geometry, vol. 56, pp. 189-234, 2000.

[16] Z. Guan, "Curvature on the Hermitian symmetric spaces," Acta Mathematica Sinica, vol. 4, no. 3, pp. 270-283, 1988.

[17] J. Cheeger and D. G. Ebin, Comparison Theorems in Riemannian Geometry, North-Holand, 1975.

[18] W. Ding and G. Tian, "Kähler-Einstein metrics and the generalized Futaki invariant," Inventiones Mathematicae, vol. 110, no. 1, pp. 315-335, 1992. 


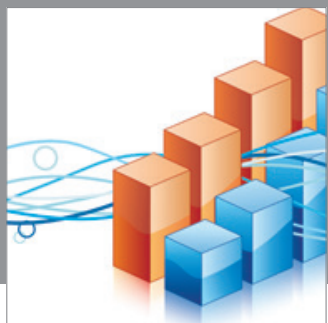

Advances in

Operations Research

mansans

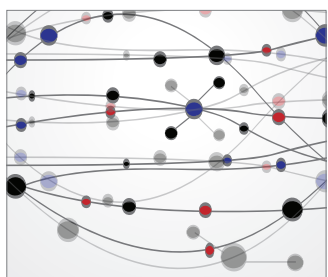

The Scientific World Journal
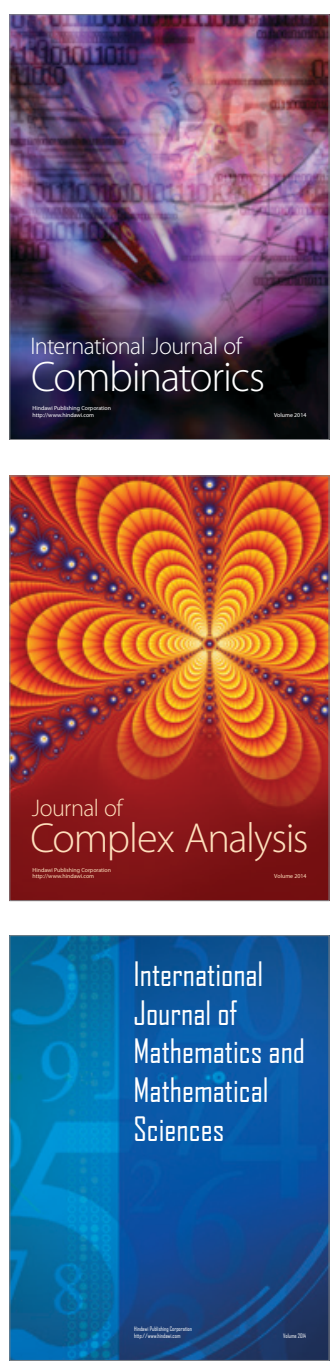
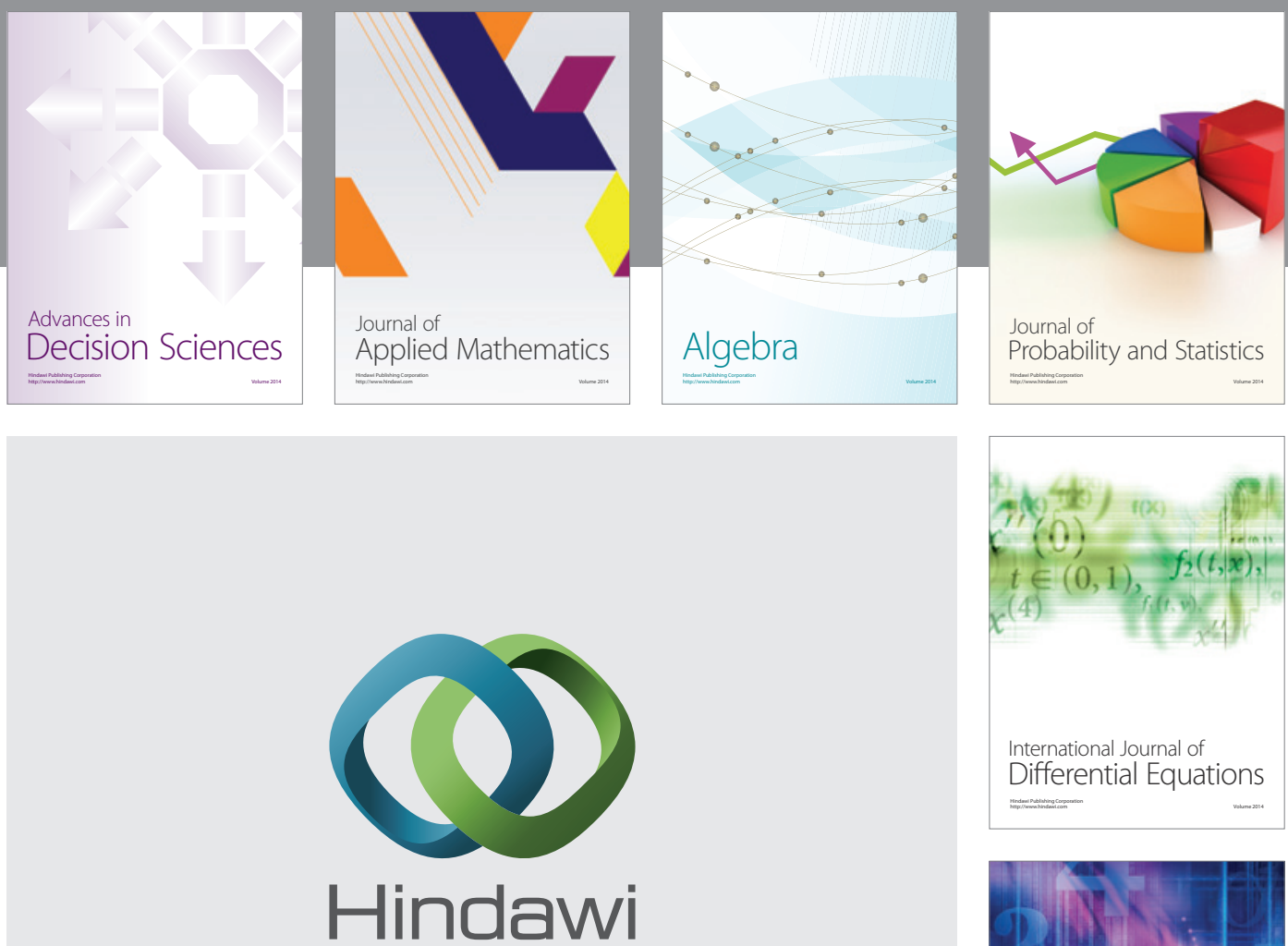

Submit your manuscripts at http://www.hindawi.com
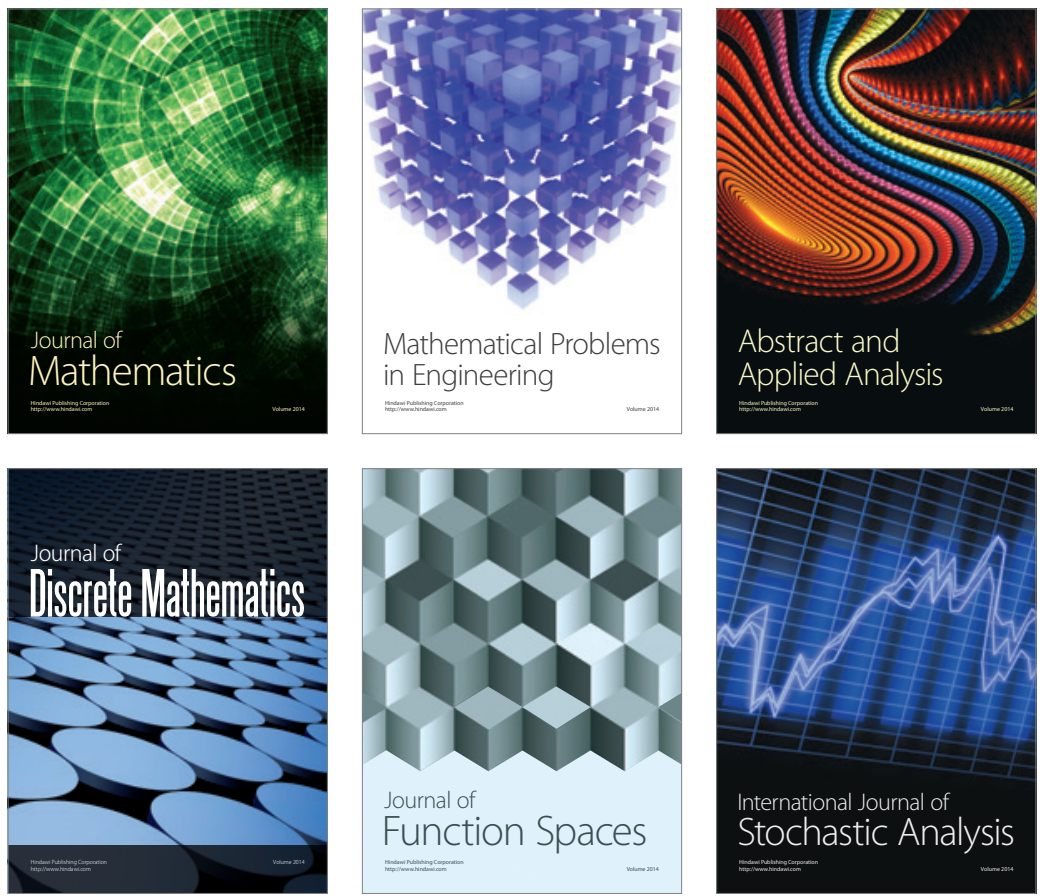

Journal of

Function Spaces

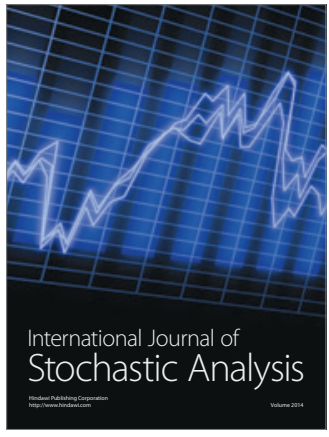

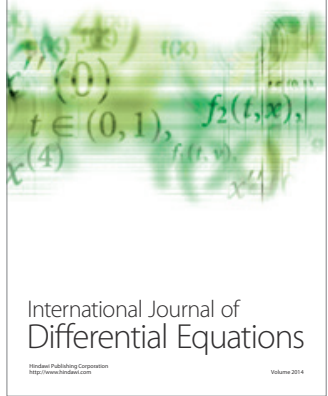
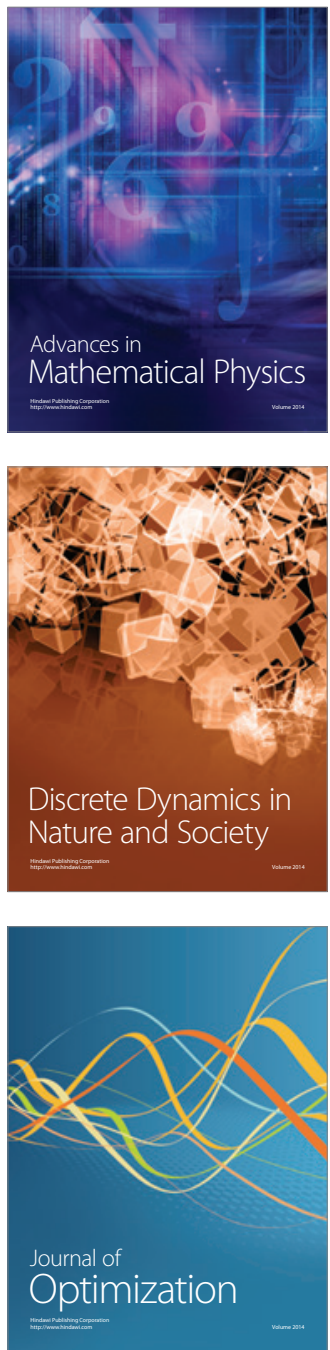